\title{
Le Sepik : société et production matérielle
}

Introduction

\section{Christian Kaufmann, Philippe Peltier et Markus Schindlbeck}

\section{(2) OpenEdition Journals}

Édition électronique

URL : http://journals.openedition.org/jso/8010

DOI : $10.4000 /$ jso.8010

ISSN : $1760-7256$

Éditeur

Société des océanistes

Édition imprimée

Date de publication : 15 juillet 2018

Pagination : 5-14

ISBN : 978-2-85430-135-9

ISSN : 0300-953x

Référence électronique

Christian Kaufmann, Philippe Peltier et Markus Schindlbeck, «Le Sepik : société et production matérielle », Journal de la Société des Océanistes [En ligne], 146 | 2018, mis en ligne le 15 juillet 2018, consulté le 24 septembre 2020. URL : http://journals.openedition.org/jso/8010 ; DOI : https://doi.org/ $10.4000 / j s 0.8010$ 


\section{Introduction}

par

\section{Christian KAUFMANN ${ }^{*}$, Philippe PELTIER ${ }^{* *}$ et Markus SCHINDLBECK ${ }^{* * *}$}

Dès sa découverte, à la fin du XIX siècle, le nord de la vallée du Sepik fut reconnu comme un des lieux où la création artistique semblait inépuisable. Les cultures installées sur les berges du fleuve et ses affluents produisaient des objets et des architectures qui provoquaient l'étonnement par leur impressionnante diversité formelle. Cette créativité a donné naissance à de nombreuses études. Des études qui ne seront jamais closes, tant la matière est riche. Les articles réunis ici en sont la preuve. Dans leur grande majorité, ils furent d'abord présentés lors du colloque organisé par le musée du quai Branly-Jacques Chirac à l'automne 2015. Ce colloque fut l'un des points d'orgue d'un projet commencé quelques années plus tôt et dont la principale manifestation fut l'exposition Sepik présentée à Berlin, Zurich et Paris en 2015-2016. Elle réunissait quelque 230 œuvres originaires de la zone marécageuse et des régions limitrophes, comprises entre l'embouchure du fleuve et l'April River.

Réduire cette zone à deux cents sculptures est un exercice difficile, redouté et forcément partiel tant la vallée du Sepik présente une marqueterie de langues et de cultures. Afin de déjouer le risque réducteur de toute exposition sur le Sepik, le visiteur était convié à parcourir différents lieux d'un village fictif. Cette déambulation était prétexte à la découverte progressive des objets d'une maison familiale vers ceux conservés dans les maisons des hommes pour finir sur ceux utilisés lors de cérémonies fastueuses. Cet artifice de présentation présupposait la présence d'une trame culturelle unissant tous les groupes de la vallée. La question d'une culture Sepik hante la recherche depuis son origine. L'existence d'objets dont les formes et les usages semblent infinis mais que présentent un je-ne-saisquoi de commun, un certain air de famille, servit de déclencheur à cette interrogation. Les objets collectés ou vus dans les villages soulevèrent, dès les premières publications, la redoutable question des différences culturelles et des frontières entre les groupes. L'artifice du parcours muséographique tenait pour acquis qu'il existait bien des traits communs ou, pour reprendre le néologisme anglais, une Sepikeness, un sentiment qui unit tous les groupes quelles que soient les formes spécifiques mises en ouvre dans les sculptures.

L'histoire de l'exploration et de la réflexion européenne sur les cultures - ou la culture - Sepik est donc un passage obligé des recherches actuelles. Si les contributions de ce numéro évoquent souvent les analyses développées au cours du siècle dernier, elles élargissent cependant notre connaissance en interprétant les objets à partir de cas précis. Cette interprétation ne repose plus seulement sur le rôle des artistes, de l'iconographie ou de l'étude des matériaux mais sur la façon dont les objets se constituent via les effets quils produisent lors de leur usage, que ce soit à travers leur apparition puis disparition au cours de rituels ou d'événements fortuits. Les contributions du présent numéro rendent compte largement des effets de la mondialisation, une mondialisation qui suscite des interrogations sur la transformation des sociétés et la perte d'un savoir, que celui-ci soit matériel ou immatériel. Paradoxalement, cette perte de connaissances a entrainé un retour aux collections conservées dans de nombreuses institutions à travers le monde. Ici encore, l'histoire est indispensable à l'analyse, mais cette lecture historique s'inscrit dans de nouveaux rapports, complexes autant que dialectiques avec les communautés d'origine.

L'espace géographique concerné ici dépasse celui retenu pour l'exposition : il inclut une partie du Haut Sepik (au-delà de l'April River) ou la zone des collines qui s'étend entre le fleuve et la côte. Cette extension géographique de la vallée à ses abords n'a rien d'une fuite en avant; elle doit s'entendre comme une étape qui, en élargissant les comparaisons, permet d'accroître notre connaissance des cultures qui se sont déployées au cours des derniers millénaires dans la vallée.

\footnotetext{
Chercheur honoraire, associé au Sainsbury Research Unit, University of East Anglia, Norwich, UK, chrkauf@yahoo.com

** Ancien conservateur général du patrimoine, Paris, musée du quai Branly-Jacques-Chirac, peltier.ph@orange.fr

*** Ancien conservateur, responsable du département du Pacific Sud à l'Ethnologisches Museum, Staatliche Museen zu Berlin, m.schindlbeck@outlook.de
} 


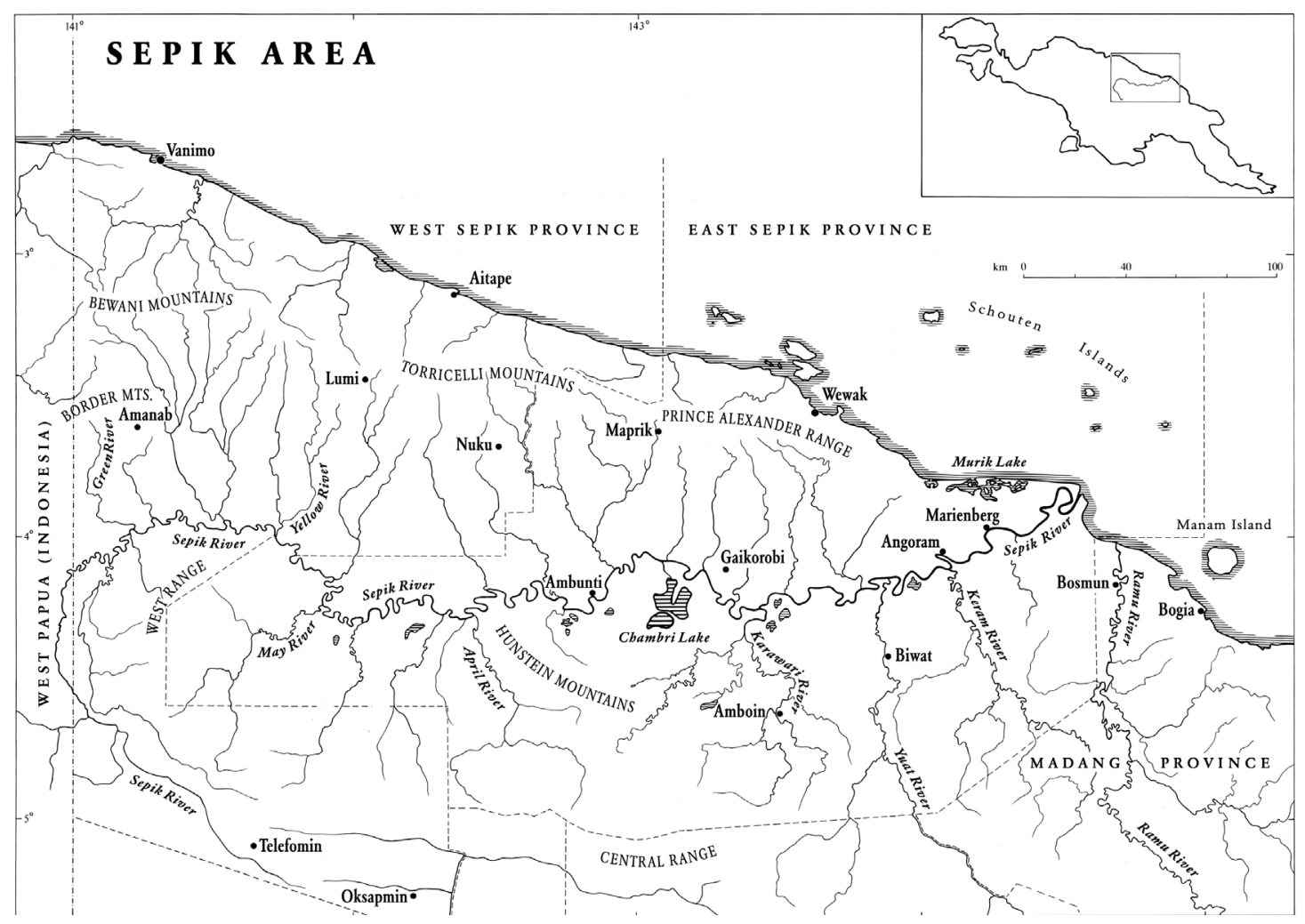

CARTE 1. - Région du Sepik avec les principaux centres administratifs et trois centres régionaux : Gaikorobi, Bosmun, Biwat (dessinée par Rudolf Zimmermann, Bâle, avec l'aimable autorisation du département Océanie, Museum der Kulturen Basel)

\section{Histoire de la recherche et des collections}

La référence constante à l'histoire nécessite de revenir brièvement sur quelques-uns de ses éléments les plus significatifs. Tout commence un jour de 1885, quand Otto Finsch et le capitaine Eduard Dallmann, accompagnés de marins allemands et indonésiens, découvrent l'embouchure du Sepik. Ils remontent le fleuve sur quelques kilomètres. Plusieurs voyages d'exploration suivirent. En 1886, le voyage de l'Ottilie est à l'origine de l'arrivée des premiers objets en Occident. Il faudra cependant attendre les années précédant la Grande Guerre pour que soient publiées des études dévolues à la culture matérielle. En 1910, paraît le livre d'Otto Schlaginhaufen sur la collection réunie lors de son voyage en 1909 pour le compte du musée de Dresde (Schlaginhaufen, 1910). En 1913, Otto Reche, qui participa à l'expédition organisée par le musée de Hambourg sous l'autorité de Friedrich Fülleborn, publiait à son tour ce qui reste une somme : sur presque cinq cents pages, il décrit une centaine d'objets sur les neuf cents collectés par l'expédition entre le 22 mai et le 4 juin 1909 (Reche, 1913). À cet ensemble, il adjoint de nombreuses pièces du Sepik déjà présentes dans les musées allemands. Pour qui s'intéresse aux cultures du Sepik, ces deux livres restent incontournables. Ils présentent non seulement les premières des- criptions des villages et de leurs habitants mais dressent un répertoire de certains objets en usage sur le fleuve ${ }^{1}$.

Otto Reche fut le premier à proposer un découpage en quatre zones stylistiques quil réduit cependant en trois zones culturelles (Reche, 1913: 475). Ce découpage est toujours utilisé ; ses frontières furent changées au fur et à mesure que la connaissance du fleuve s'étendait vers l'amont. Tout au long de son texte, Reche remarque que certains objets ont une réelle valeur artistique. Cette remarque va à l'encontre de l'idée répandue de nos jours que les ethnographes du début du $\mathrm{xx}^{\mathrm{e}}$ siècle étaient indifférents à la valeur artistique des objets. Il suffit de lire les premiers textes pour comprendre combien cette idée est fausse. Ainsi l'article de von Luschan publié dès 1911 dans le Baessler-Archiv. Il y recommandait rien moins que la mise en place d'un programme d'une étude de terrain sur l'art du Sepik (von Luschan, 1911).

Cet article était indiscutablement connu des participants à l'expédition pluridisciplinaire organisée en 1912-13 par le musée d'ethnographie de Berlin. Sous la direction d'Artur Stollé, l'équipe était composée, entre autres, de Carl Ledermann, botaniste, de Walter Behrmann, géographe, d'Adolf Roesicke et de Richard Thurnwald, tous les deux chargés de l'ethnographie. Elle séjourna plusieurs mois dans la vallée, Thurnwald prolongeant son séjour jusqu'en 1914. Hélas, la Première Guerre mondiale ne permit pas

1. Cet inventaire est certes incomplet. Les sociétés vivant le long des affluents du Sepik ne sont pas représentées et certains types d'objets ne furent probablement pas collectés. Aussi passionnante qu'elle soit, cette question de la représentativité est trop complexe pour être développée ici. 
la publication du voyage. Les collections, ou ce qu'il en restait après les vicissitudes de l'histoire, ne furent publiées que cinquante ans plus tard quand les objets retrouvés furent transférés dans le nouveau bâtiment du musée de Dahlem (Kelm, 1966 pour les vol. 1-2, 1968 pour le vol. 3) ${ }^{2}$. L'important journal de voyage de Roesicke (il devait mourir peu après la Première Guerre mondiale) fut publié quelques cent ans plus tard (Schindlbeck, 2015; Roesicke, 1914). Nous sommes cependant redevables à Behrmann non seulement d'une carte remarquable de précision (Behrmann, 1917-1924) et d'un livre (Behrmann, 1922), dans lequel il propose, suite aux travaux de Roesicke, une classification des groupes culturels en fonction des formes de l'architecture cérémonielle.

Dès les premières années du Xxe siècle, le Sepik suscita un intérêt qui dépassa les limites de la puissance impériale allemande et son projet colonial. Â la suite de celle de George Dorsey, une expédition d'envergure fut organisée par Albert B. Lewis alors assistant conservateur au musée d'Histoire naturelle de Chicago. Après un long périple dans le Pacifique et plusieurs arrêts sur la côte nord, son bateau remonta le fleuve sur 465 kilomètres entre le 9 et le 16 août 1910. Lewis acheta massivement lui aussi des objets dans les villages. À son retour, il publia quelques études sur ses collections et organisa leur installation dans les nouvelles galeries du Field Museum. Là encore, l'histoire se répète puisque son journal de voyage ne sera édité qu’en 1998 (Welsch, 1998).

Cette rapide présentation de trois expéditions organisées par des institutions muséales permet de souligner un paradoxe : alors que ces institutions conservent pour ne pas dire accumulent! - un nombre considérable d'objets, les aléas de l'histoire firent que ces premières collections restèrent peu connues. Ces objets ainsi que les informations disponibles sur leurs origines permettaient cependant de donner une image relativement complète de la production matérielle de la région. Comme le montrent plusieurs articles présentés ici, l'exploitation de la documentation accumulée au cours de ces expéditions permet de redonner vie à certains objets.

La vie sur le fleuve ne fut pas perturbée par la Première Guerre mondiale. À long terme, le changement vint du remplacement de l'administration allemande par l'administration australienne. Cette dernière fut cependant lente à se mettre en place. Après la guerre, les villages commencèrent à se vider et de nombreux jeunes hommes partirent travailler sur les plantations. Ils en revinrent avec de nouvelles connaissances, l'usage d'une nouvelle langue de communication - le pidgin de Nouvelle-Guinée, aujourd'hui appelé Tok Pisin - et de nouvelles richesses. Ces départs eurent probablement pour effet de ralentir la production d'objets. Quoi qu'il en soit, les collectes se poursuivirent. L'époque n'était plus aux grandes expéditions scientifiques mais plutôt aux voyages organisés par de riches voyageurs ou quelques colons, dont les acqui- sitions enrichirent les collections des musées. Ainsi le British Museum reçut un don de Lord Moyne (voir Bolton infra), l'Australian Museum acheta des objets à Wauchope, un planteur installé dans la baie de Hansa (voir Bolton, Barlow et Lipset) et Axel Bojsen-Moeller, donna au musée de Copenhague quelque 800 objets collectés lors de son voyage entre 1933 et 1934. Quant au Musée de l'homme, il reçut, à la veille de la Seconde Guerre mondiale, le dépôt des objets collectés lors de la croisière entreprise entre 1934 et 1936 par de riches amateurs français sur le bateau La Korrigane (Coiffier, 2001). Plus original est le cas de la War Collection déposée de nos jours au Victoria Museum de Melbourne, puisqu'elle est constituée d'objets acquis ou saisis par les Australiens lors de leur prise de contrôle de la partie allemande de la Nouvelle-Guinée (Craig et al., 2015).

Durant cette période un corpus important de prises de vue photographiques et cinématographiques de la vie villageoise fut réalisé tout au long du Sepik. Pour mémoire citons les travaux de la Crane Pacific Expedition de Chicago en 1928-1929 et ceux, en 1930 de Felix Speiser et Heini Hediger. Ces deux derniers chercheurs constituèrent une collection pour le musée d'ethnographie de Bâle et eurent la chance de filmer un moment de l'initiation des garçons. Leur film montre comment des masques-crocodiles avalent des jeunes garçons et inscrivent sur leur peau des scarifications qui témoignent de leur passage dans le monde ancestral et mythique

Cette période de l'entre-deux guerres est dominée par les travaux de Gregory Bateson et de Margaret Mead. Bateson ne publiera jamais d'étude sur l'art du Sepik - ses écrits sur l'art furent réservés à Bali (Coupaye, infra) - mais il rédigea des fiches très précises sur les 600 pièces qu'il collecta (Lovelace, infra). Quant à Margaret Mead, elle constitua des collections dans plusieurs régions du fleuve et publia de nombreuses études sur l'art du Sepik. En 1938, le volume d'introduction à sa monographie The Mountain Arapesh, Part 1 portait un sous-titre explicite: An importing culture (Mead, 1938). Avec cette étude, l'image du Sepik se complexifiait : les cultures ne pouvaient plus être considérées comme des isolats mais s'inscrivaient dans un processus dynamique d'échanges.

La Seconde Guerre mondiale et les années qui suivirent furent un temps de silence. Le Sepik, marqué par la présence massive des forces japonaises, soignait ses plaies. De ce moment important au regard de l'histoire, nous ne savons pas grand-chose sauf ce qu'ont bien voulu en dire, dans leurs souvenirs ou les rapports de patrouilles, les kiap, ces jeunes Australiens formés à l'administration du territoire colonial. À partir des années 50, la rivière fut à nouveau le lieu d'une intense activité de recherche. L'un des premiers collecteurs fut Paul Wirz, qui exposera, à la demande de Bühler, sa collection à Bâle en 1954. Wirz et Bühler soulignèrent alors la valeur artistique des pièces

2. Notons cependant qu’un supplément regroupant les pièces retrouvées après le transfert des collections au musée de Dahlem fut publié en 1969 (Kelm, 1969). 
(Bühler et Wirz, 1954)3. Suite à la mort soudaine de Wirz à Maprik en 1955, Bühler entreprit son premier voyage en 1955-56. Fasciné par le fleuve, il y retourna en 1959, cette fois en compagnie d'Anthony Forge (Kaufmann, 2017). Ces deux voyages marquent une étape fondamentale non seulement au regard du nombre et de l'importance des objets collectés, mais aussi des problématiques qui en naquirent. À son retour, Bühler publia une carte stylistique détaillée de la région (Bühler, 1960). Maintes fois reprise, précisée mais aussi critiquée (Craig, infra), elle reste une référence incontournable. Le voyage de Forge sur le fleuve fut à l'origine d'une étude comparative qui, publiée en 1965 (Forge, 1965), marquera de nombreux travaux ultérieurs. Dans son texte, Forge tente de cerner les traits communs entre deux sociétés voisines, les Abelam - où il mène ses propres recherches de terrain - et les Iatmul, dont les formes artistiques sont radicalement distinctes. D'une étonnante façon, Forge considère, qu'eu égard à la taille des sociétés et l'ampleur de leur production, la culture matérielle de ces deux groupes domine le Sepik (Forge, 2017 [1986] : 162). Ce faisant, il laissait dans l'ombre une grande partie des autres cultures du Sepik! On peut s'interroger sur une telle exclusion et ses conséquences. Elle traduit probablement une réalité du terrain dans les années 1960-70: en 1913, l'installation de la mission catholique sur la colline Marienberg à quelques miles de l'embouchure du fleuve, puis en 1918, des forces australiennes à Angoram dans le bas Sepik provoquèrent une transformation sociale radicale et la perte de nombreuses traditions. Depuis, le bas Sepik souffre indiscutablement d'un manque d'études, à l'inverse d'autres régions dont les traditions restent plus vivantes. On prendra pour exemple les groupes habitant dans l'Alexander Range qui ont été étudiés à différentes époques par de nombreux chercheurs (citons, entre autres, Mead, Forge, Tuzin, Roscoe, Coupaye) ou encore le rituel naven des Iatmul qui, depuis la parution du livre de Gregory Bateson en 1936, fascina les anthropologues (voir à ce sujet Houseman \& Severi, 1994). Pourtant, comme le démontrent les articles du présent recueil, la région du bas Sepik et celle de la côte engagent des problématiques relatives aux échanges, à l'identité et à la manifestation des ancêtres qui s'avèrent tout aussi importants pour comprendre des mécanismes fondamentaux aux société du Sepik ( $c f$. Barlow, von Poser mais aussi Bolton infra).

Les années 1950-60 furent elles aussi celles d'intenses collectes, qui ne se limitèrent pas à l'action suisse! Citons, entre autres, celle entreprise entre 1953 et 1956 par Carel Groenevelt pour le musée de Rotterdam, celle de Françoise Girard pour le musée de l'Homme en 1955, celle que Jean Guiart effectua en 1961 afin de constituer un fonds de collection pour le tout nouveau musée des arts d'Afrique et d'Océanie à Paris, celles que Meinhard Schuster et Eike Haberland réunirent pour le musée de Frankfurt (Haberland et Schuster, 1964). En 1986, Schuster publiait un article important resté largement méconnu, qui traitait de la transformation des motifs sur les boucliers du Haut Sepik (Schuster, 1986) et Guiart un opuscule commandé par l'unEsCo afin de faire découvrir l'art de cette région au grand public (Guiart, 1968). Citons enfin - mais cet inventaire est loin d'être exhaustif - les travaux de Douglas Newton dans la région Kwoma/Manambu, qui se concrétisèrent dans une exposition tenue à New York en 1971 (Newton, 1971). Dans ces mêmes années, fut découverte une forme d'art jusque-là largement inconnue - même si quelques rares objets avaient atteint les collections occidentales - originaire de la région du Korewori (Haberland, 1968 ; Kaufmann, 2003). Ce fut l'ultime surprise livrée par le fleuve. Ainsi, dans les années qui précèdent l'indépendance de la Papouasie Nouvelle-Guinée en 1975, les connaissances sur la culture matérielle de la région changent de ton. Un matériel considérable, souvent témoin des transformations importantes qui ont eu lieu aussi bien dans les modes de production que dans l'iconographie ou le type d'objets, arrive en Occident et se double parfois d'informations précises sur l'usage ou la fonction des objets.

Cette effervescence trouve une conclusion temporaire dans un événement marquant mais sous-estimé : l'ouverture en janvier 1982 de la "Rockefeller Wing " au Metropolitan Museum of Art de New York dévolue aux "arts primitifs». À cette occasion, émergea l'idée d'une coopération entre New York et Bâle, qui prit la forme d'un colloque sur le Sepik qui se tint à Bâle en 1984. De nombreux spécialistes, pour ne pas dire tous ceux qui avaient alors travaillé dans la vallée, étaient présents. Le thème de la conférence, "Tradition et changement", marque indiscutablement la prise en compte de nouvelles problématiques mais aussi le souci d'apporter, par le biais d'études anthropologiques, une réponse aux problèmes posés aux villageois, dix ans après l'indépendance, du lent passage à la modernité (Lutkehaus et al., 1990).

La conférence de Bâle fut suivie en 1986 d'un second colloque qui se tint à Mijas en Espagne. Il fut organisé par Anthony Forge. L'anthropologie et la dynamique des cultures étaient au centre des discussions. Hélas, les actes de ce colloque ne virent jamais le jour. L'introduction qu'écrivit Forge pour cette publication vient, elle, d'être éditée, plus de trente ans après la tenue de la conférence (Forge, 2017). Ce texte est tout aussi stimulant que l'article du même auteur publié en 1965. Forge remarque que les "activités de production " dans le Sepik sont effectuées par les hommes et les femmes de manière équilibrée. Aux premiers revient la mise en œuvre de rituels centrés sur les maisons des hommes, aux secondes la production de la nourriture et l'éducation des jeunes enfants ${ }^{4}$. Il note :

3. Cette collection est conservée de nos jours au Tropen Museum d'Amsterdam. Le musée publia le texte de Wirz en 1959.

4. Forge semble ignorer, entre autres, le rôle des hommes dans la guerre! Mais déjà dans les années 1960, celle-ci tient plus du souvenir que de la réalité. 
"The Sepik is therefore arguably the most coherent area of the lowlands in the whole island where the old patterns of interaction and the production of ritual and its accessories remained, if not intact until the 1960s, at least of considerable importance to the lives of its inhabitants who had retained everywhere an excellent knowledge of ritual and the details of its performance. " (Forge, 2017 [1986] : 156)

Ainsi, pour Forge, les études sur les rituels entendus dans leurs formes anciennes sont toujours possibles dans les années 1960-70. Pourtant, à la date de la rédaction de ce texte (1986), les sociétés du Sepik sont en pleine mutation. Les années 1980 et 1990 seront marquées par une migration plus pérenne et de plus en plus importante des villageois vers les centres urbains. La région se vide lentement mais inexorablement. La question de la survivance de la " kastom" (la tradition), y compris dans les villages, commence à se poser. Dans un texte qui fait suite à une mission chez les Banaro - un groupe de la Keram - en 1989, Bernard Juillerat dresse le constat d'une perte de mémoire considérable, au point d'évoquer "une ethnologie historique " (Juillerat, 1993).

\section{Contributions}

Dans les dernières années $\mathrm{du} \mathrm{xx}^{\mathrm{e}}$ siècle, le Sepik a bien basculé dans un autre monde. Celui de la modernité. Les textes réunis ici le confirment. Tous ou presque parlent des changements sociaux et de l'adaptation au monde contemporain. Aucun des textes ne trahit des signes de nostalgie face à une époque disparue. L'ethnologie a traversé le miroir du temps. À l'étude des formes sociales anciennes s'est substituée l'analyse des phénomènes contemporains. Mais ce passage à la modernité est aussi un défi lancé aux musées. L'étude des collections majoritairement constituées dans la première moitié du siècle dernier implique que les nouvelles approches prennent en compte l'histoire. Les contributions que l'on va lire ici sont regroupées sous trois têtes de chapitres dévolus respectivement aux rituels, aux collections et aux défis face au futur. Un quatrième ensemble permet l'élargissement des axes de réflexion à de nouveaux champs et à des régions limitrophes à la vallée.

\section{Rituels et matérialité}

Une des figures mythiques présente dans de nombreuses sociétés du bas Sepik est celle connue à Murik sous le nom de Nzari (elle peut prendre, selon les groupes, d'autres noms). Selon certains mythes, elle descendit le fleuve, selon d'autres, elle serait originaire de la côte. Tout à la fois guérisseuse et sage-femme, elle est à l'origine de nombreux aspects de la culture et du paysage, qu'elle contribua à modeler.

Jusque récemment, la figure de Nzari a été sousestimée. Elle est pourtant incontournable car elle permet de ré-évaluer les rapports entre les sexes mais aussi d'appréhender l'étonnant pouvoir symbolique des objets utilisés ou fabriqués par les femmes. Dans son texte, K. Barlow montre que le pouvoir des femmes dans la région du lac Murik repose sur le don de nourriture à l'occasion de cérémonies organisées par les hommes, don qui permet aux époux de gagner en prestige. Ce pouvoir des femmes repose aussi sur la fabrication de paniers tressés dont les motifs complexes sont des marqueurs identitaires avant de devenir des objets supports de mémoire. Quant à A. von Poser, elle analyse les poteries dont le secret de fabrication fut donné par Nzari aux femmes de Bosmun. Ces poteries sont l'expression de l'identité féminine; les femmes y transforment la fécule de sagou en une gelée comestible, opération mystérieuse de coagulation liée métaphoriquement à la gestation de l'enfant.

Ces marqueurs identitaires se modifient face à la modernité. K. Barlow décrit le processus de transformation à la fois dans les échanges traditionnels entre groupes voisins mais aussi à l'occasion du passage récent au marché touristique. A. von Poser, en s'appuyant sur Gell, montre combien l'investissement symbolique s'est récemment déplacé de la fabrication des objets à d'autres productions matérielles. À Bosmun, cet investissement affectif semble cependant être plus sensible aux changements contemporains que dans la région du lac Murik: le savoir lié à la fabrication des poteries n'est plus connu des jeunes générations alors que les paniers tressés du lac Murik, toujours fabriqués aujourd'hui, jouent un rôle central lors des cérémonies funéraires ou des prises de grade mais aussi dans l'économie locale.

La figure mythique de Nzari, héroïne fondatrice de nombreux aspects de la culture, continue à hanter le bas Sepik. À l'origine d'une palette de traits sociaux mais aussi d'objets produits par les femmes, cette figure tutélaire permet d'analyser les processus de résistance ou d'adaptation aux changements survenus dans des sociétés géographiquement et culturellement proches mais dont les milieux écologiques différents modèlent l'économie et les rapports sociaux.

N. Garnier et E. Silverman traitent d'une question lancinante de l'anthropologie du Sepik : le savoir des femmes sur les secrets des hommes. Pour N. Garnier, les femmes en savent beaucoup plus qu'elles ne le disent. Tout relève du paradoxe puisque tout le monde sait que tout le monde sait sans vouloir l'avouer. Le secret serait une fiction. Les femmes voient effectivement beaucoup d'objets d'hommes. N. Garnier et E. Silverman déplacent ainsi la dynamique des rapports hommes/femmes. Par-delà cette séparation des sexes, se pose le problème du savoir face aux choses vues. Voir est-il un fait suffisant? L'important n'est-il pas de connaître les noms et les histoires attachés aux objets? Pour N. Garnier, cette indifférence des femmes aux secrets des hommes ne serait pas due aux changements sociaux récents. Cette question reste ouverte à débat.

Un second ensemble d'articles traite plus directement de la transformation face aux exigences des églises évangéliques. Les sociétés traditionnelles étaient dominées par les esprits liés au territoire (Vávrová et Telban, infra). Ces esprits peuvent être des résidents perma- 


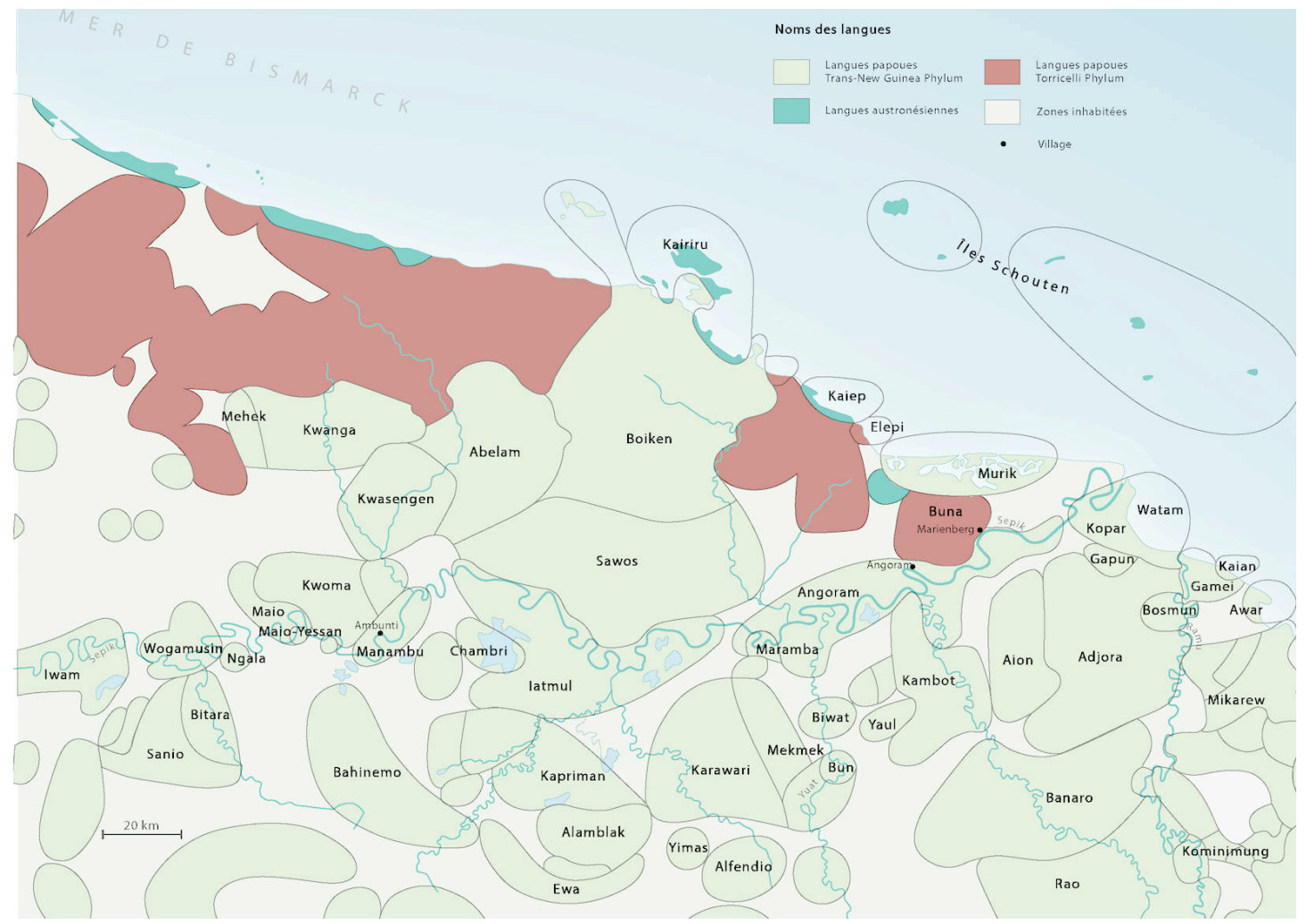

CARTe 2. - Carte des langues de la région Sepik-Ramu (avec l'aimable autorisation du musée du quai Branly, Paris, () Thierry Renard)

nents alors que d'autres migrent avec les groupes. Ils apparaissent en rêve et leurs figures peuvent alors être sculptées puis installées dans la maison des hommes où, au cours d'une cérémonie, elles reçoivent un nom. Borut Telban montre ainsi combien rêve et réalité relèvent d'un même aspect, qu'une image-esprit ressort tout à la fois du domaine du rêve et du monde réel. Cette appartenance à deux espaces leur confere un pouvoir de prédiction. Comme l'indique B. Telban, ces esprits, comme tout objet - même moderne - ne peuvent exister qu'après avoir reçu un nom. Pour un homme du Sepik, un objet sans nom est une coquille vide. Nommer, c'est faire exister.

Cette conceptualisation du monde a un effet pour le moins inattendu quand la croyance au Dieu de l'Occident est adoptée (Vávrová, infra). Malgré la conversion des villages au mouvement charismatique, ces esprits qui appartiennent au monde du rêve sont toujours présents. Or, le dieu occidental est tout à la fois exclusif - joignable grâce aux téléphones portables, son numéro n'est connu que de très peu de gens - et sans territoire précis. Détenteur lui aussi du futur, sa capacité à prédire l'avenir est cependant parfois remis en question. Une cure charismatique repose sur la prière, celle des guérisseurs sur l'extraction d'un objet, qui est la preuve visible de l'origine de la maladie. Son extraction est indispensable à la preuve du succès de l'opération. Le dieu de l'occident souffre d'une forme d'incomplétude face aux esprits : il ne fournit aucun élément tangible.
Ce même pouvoir des objets est au centre de l'étude de T. Bartole qui analyse l'action d'un mouvement charismatique promettant des temps futurs radieux. La réalisation de ce futur divin se joue autour de l'apparition puis de la possession d'une statue de la Vierge, qui, dans le cas présent, n'apparaîtra pas. Cette absence est interprétée - et l'on peut penser qu'il en fut parfois de même dans les sociétés traditionnelles - comme le refus d'un don. Or, ce don, qui est une technique au sens maussien du terme, doit être périodiquement renouvelé en touchant la statue afin d'être effectif. Ce pouvoir du touché donne à l'auteur l'occasion d'analyser finement une nouvelle pratique (peut-on parler ici de rite ?) répandu dans le Sepik : celui du serrement de main entre des groupes villageois afin de clore des conflits.

Ces contributions permettent de repenser la place de l'objet dans la société. Non plus par sa matérialité la plus banale mais par son pouvoir qui s'inscrit dans un rapport dynamique entre l'objet et son utilisateur par le fait de nommer, de voir et de toucher. Ces actions sont indispensables à la reconnaissance de l'objet. Elles permettent de saisir qu'un objet ne se limite pas à sa matière car il ne peut s'affirmer sans faire l'économie du rapport au corps ou à l'esprit. Un objet, pour exister et faire valoir son pouvoir, ne peut le faire sans l'aide des sens et du langage. R. LambertBretière introduit quant à elle un tout autre aspect de la modernité. Elle montre les effets encourus par la société kwoma suite à la disparition des chants rituels liés aux cérémonies des ignames. Ces chants rituels, 
connus de quelques hommes, sont rarement produits. Lors de l'un de ses séjours, l'auteur a eu la chance d'assister à une telle performance. La description de l'accumulation des appareils d'enregistrement posés sur une table devant la maison des hommes où a lieu la cérémonie dit, à elle seule, la crainte, reconnue par les Kwoma, de l'effacement et le souci de transmettre les savoirs. Dans cette scène se dessine aussi en creux la position des femmes chercheuses dans la société en tant que femme elle ne pourra avoir accès au lieu du rituel - mais aussi la place du secret - les chants ne pouvant être publiés. L'auteur montre aussi combien la modernité bouscule le rapport de la société à l'espace et au temps. Effectivement, les hommes qui connaissent ces chants vivent dans les centres urbains et la mise en œuvre de cérémonies dépend de la temporalité des villes. Un autre espace, aussi bien mental que physique, se dessine.

\section{Collectionner et exposer}

Au cours de ces dernières décennies, l'histoire des collections a fait l'objet d'une attention grandissante. Les conservateurs de musées, souvent rompus aux recherches de terrain, en furent les chevilles ouvrières. Afin de dresser une histoire des voyages et des collectes, ils entamèrent l'exploration des archives. Cette attention à l'histoire des objets coïncida peu ou prou avec l'émergence de l'idée selon laquelle les musées peuvent être considérés comme des "zones de contacts", des lieux-ressources pour les communautés. Dans leurs travaux, L. Bolton et C. Hellmich montrent, à partir de cas d'étude précis, toute la richesse émergeant de la conjugaison de ces deux approches.

Trois figures féminines sont le point de départ de l'étude de L. Bolton. Elles sont conservées dans trois musées différents où les inventaires les ont attribuées au bas Sepik sans préciser ni leur usage ni leur sens. La recherche sur leur origine plus précise et leur fonction donne lieu à une longue enquête où se conjuguent dépouillement d'archives et retour sur le terrain. Aux archives de préciser l'origine des objets sur fond d'histoire des collectes, au terrain celui de préciser leur fonction. Or, la distance temporelle qui sépare la collecte de l'enquête de terrain - plusieurs décennies soulève bien évidemment la question de la reconnaissance et de l'interprétation, les informateurs ayant une certaine tendance à attribuer à d'autres groupes les objets qu'ils ne reconnaissent pas. Christina Hellmich, quant à elle, part pour son étude d'un masque collecté dans le village de Masendenei et conservé au De Young Museum à San Francisco. Ce masque est un cas d'école. D'après différentes sources, il est l'un des objets les plus anciens du Sepik. Or, depuis sa sortie du territoire, il a été remplacé par quatre autres masques qui présentent chacun des variations. Les villageois les considèrent tous comme authentique alors que les spécialistes occidentaux n'y voient que de simples copies. Cet exemple démontre la relativité de la notion d'authenticité. Pour les villageois, elle repose sur le fait de recevoir un nom et de présenter une iconographie en accord avec la tradition, pour l'Occident, c'est l'ancienneté qui prime.

Ces deux derniers articles soulèvent la question d'un savoir contemporain sur des objets anciens. Ils apportent un nouvel éclairage sur le rôle de la mémoire. Comme le remarqua un artiste iatmul lors de sa visite récente à San Francisco, lorsqu'il s'agit de copier un objet, avoir la mémoire des récits mythiques le concernant est plus important que d'avoir l'objet en modèle. Ce rôle de la mémoire engage bien évidemment la question de l'invention des formes, une invention dont on peut penser qu'elle était déjà à l'œuvre dans les temps anciens. Quoi qu'il en soit, ce processus est en accord avec le savoir du moment, un savoir dont l'authenticité, face aux interprétations passées, ne peut pas être remise en cause.

Le savoir sur les objets n'est ainsi jamais figé dans le temps. Il nait d'un lent processus d'accumulation. Ce savoir lié à un instant donné pose le problème de l'exploitation des sources afin de cumuler et de traiter les données recueillies à différentes époques par différentes personnes pour les fondre, si possible, dans un ensemble cohérent. Cet exercice périlleux mais indispensable fut entrepris lors de la constitution du Basel Iatmul Catalogue of Objects. Christin Kocher Schmid en rend compte ici. Quant au catalogue de la collection constituée par Gregory Bateson, il est étudié par Antonia Lovelace. Les deux articles laissent entrevoir le gain considérable qu'il y aurait à confronter et à synthétiser ces travaux qui n'ont connu jusqu'à nos jours, hélas, qu'une diffusion restreinte.

On pourrait espérer que le développement technologique récent faciliterait la publicité de ces travaux. Or, la mise en ligne des données se heurte à de nombreux impératifs : légaux comme le droit sur les images, scientifiques comme la hiérarchisation des informations, sociaux comme l'accessibilité par tous les membres d'une société à des objets dont l'usage est toujours secret. Cette mise en ligne dépend de la volonté des musées de travailler en réseau. Une mise en réseau n'est d'ailleurs pas le seul problème tant les recherches sur les collections dépendent du type de collections. Il existe une énorme différence entre une collection récente regroupant peu de pièces anciennes et une collection ancienne où les objets se comptent par milliers. L'intérêt d'une petite collection réside dans le fait que chaque objet peut être soumis à une lecture intensive et ouverte, celle de grandes collections que des recherches extensives recouvrant tous les aspects d'une société peuvent lui être consacrées.

On en vient ainsi à l'interrogation sensible mais inévitable sur les collectes contemporaines. David Lipset, en forme de réponse à une critique souvent émise - le pillage et le respect des interlocuteurs locaux - expose ce qu'est, pour lui, une collecte de type postcoloniale. Elle se fonde sur la coopération entre chercheurs et informateurs et suppose une connaissance précise du terrain et des réseaux sociaux locaux. Il montre, sans fard, que les collectes sont suspendues aux aléas de la vie : l'article raconte son séjour sur le terrain avec K. Barlow et L. Bolton afin d'étudier les collections de 
la région du lac Murik conservées à l'Australian Museum de Sydney. Il n'insiste pas sur les changements mais l'on comprendra évidemment que ce ne sont pas forcément les objets recherchés au début du siècle qui, de nos jours, attirent l'attention des collecteurs.

\section{Vers le futur}

Cette dernière remarque engage l'enjeu de la politique d'acquisition des musées. Doit-elle se tourner vers le passé, comme c'est souvent la tentation, en n'acquérant que des objets anciens ou traditionnels ou bien doit-elle s'ouvrir aux expressions contemporaines? L'étude d'E. Silverman porte justement sur des objets inventés ces dernières décennies: des peintures "modernes " exécutées sur les carrosseries des camions ou encore les objets souvenirs produits pour les touristes. Bien que vues et photographiées par de nombreux voyageurs, les peintures sur les camions ont peu attiré l'attention des chercheurs. Elles ont été longtemps considérées comme trop anecdotiques ou trop « kitch». Nos catégories pervertissent bien souvent notre perception des choses. L'auteur part d'un constat plus ouvert : au lieu de juger de la valeur de ces productions, il les analyse comme relevant d'une nouvelle dynamique de la pensée. En revenant sur la question des niveaux de sens, il montre que la participation au monde moderne passe par l'inclusion de signes qui renvoient à l'histoire locale ancienne. Si, en peignant des paysages idylliques, les Iatmul ne s'adressent pas directement aux conflits sociaux ou environnementaux nés des temps modernes, leurs œuvres traduisent cependant une critique de la modernité.

Le texte d'E. Silverman revient ainsi sur la question lancinante de la migration des sens, une question réexaminée ici par Brigitta Hauser-Schäublin. Afin de démontrer les problèmes liés à l'interprétation et à l'adaptation des signes dans le temps et dans l'espace, elle prend pour point de départ le cas des patola, ces tissus indiens importés au XVIII ${ }^{\mathrm{e}}$ siècle en Indonésie. L'exemple peut paraitre anachronique; il permet cependant de mesurer la distance entre l'autrefois et le maintenant et ouvre à une réflexion sur les méthodes d'analyse. Bien évidemment, mais de façon transversale si l'on ose dire, l'auteur repose la question de l'authenticité des objets. Cette notion ne saurait s'appliquer à la dynamique contemporaine des sociétés. Les objets anciens, de nos jours connus grâce à la circulation accélérée de l'information, font l'objet de réappropriations lors de festivals urbains qui se multiplient dans le Sepik comme dans le reste du Pacifique. Lors de ces monstrations, la question n'est plus celle de leur authenticité mais de leur force de représentation identitaire.

\section{Au-delà du Sepik}

Les contributions réunies dans la dernière partie dépassent tout à la fois les frontières de la vallée et ouvrent à des domaines souvent négligés. Il en est ainsi de la musique. Le domaine est abordé par Raymond Ammann, musicologue connu pour ses travaux au Vanuatu et en Nouvelle-Calédonie. Sa contribution est centrée sur trois instruments du Sepik: les tambours-sabliers, les tambours à fente et les flûtes traversières. R. Ammann rappelle que les recherches sur la musique se comptent sur les doigts d'une main. Cette absence est d'autant plus regrettable que l'étude de la musique, ici à partir du seul cas iatmul, montre la cohérence du système de pensée : la structure répétitive tout comme l'alternance des motifs musicaux peuvent être vus comme un miroir des structures dualistes présentes dans toutes les sociétés de la vallée. La musique serait, au même titre que la structure sociale, un élément essentiel pour définir la "Sepikness».

Si l'article de R. Ammann souligne le gain obtenu à inscrire l'étude de la musique dans les programmes de recherche, Barry Craig démontre, lui, que l'élargissement du corpus des objets remet en cause l'idéemême des zones stylistiques. La critique de B. Craig est radicale : après avoir remarqué que la carte qui sert de référence fut établie par Alfred Bühler en prenant pour étalon la grande sculpture (Bühler, 1960)5, il montre que les zones stylistiques varient en fonction des catégories d'objet. L'étude publiée ici est limitée aux types d'objet les plus susceptibles d'être échangés, comme les étuis péniens. Or, ces derniers, outre d'être explicitement associés au genre des individus, sont aussi des marqueurs de l'identité d'un groupe. Objets intimes, on peut se demander s'ils sont présents dans les réseaux d'échange ou si seuls leurs motifs circulent. Se pose alors la question des critères représentatifs et de leur pertinence. Comme le note B. Craig, à force de raffiner l'analyse, on risque d'aboutir à un vaste méli-mélo dans lequel il est impossible de se retrouver.

L'article de Markus Schindlbeck porte lui sur un corpus d'objets importants mais oubliés des travaux contemporains: les pierres dressées. Pourtant, l'analyse de ces objets a hanté l'histoire, les auteurs anciens y voyant tout à la fois un marqueur de l'expansion des populations et des objets liés à la chasse aux têtes. Tout en reprenant les études et les documents anciens, Schindlbeck montre que ces pierres sont associées aux migrations des groupes et à la fondation des villages. Bel exemple de l'occultation, par une lecture liée à une théorie de l'époque - qui se double ici d'un sujet aussi fascinant que sulfureux, la chasse aux têtes - d'objets identitaires liés aux ancêtres et à l'histoire des migrations des groupes locaux. Quant à Alexandra Aikhenvald, elle examine la dynamique des emprunts entre langues. Lors de son terrain, elle s'aperçoit que les $\mathrm{Ma}-$ nambu, groupe numériquement important et vivant sur le fleuve, empruntent de nombreux mots au Tok Pisin, alors que les Yalaku, qui habitent à l'intérieur des terres et dont le nombre de locuteurs est faible, n'ont que rarement recours à cette lingua franca. Pour l'auteur, ce cas d'école démontre qu'un habitat ouvert aux échanges comme celui des Manambu n'est pas le

5. L'une des applications directe fut, par exemple, l'exposition The Art of the Sepik, organisée par Allen Wardwell à l'Institut d'art de Chicago en 1971 (Wardwell, 1971). 
seul facteur déterminant à l'usage de nouveaux mots. Une langue résiste mieux dans des petits groupes, la conscience d'une perte identitaire y étant plus aiguë.

Enfin, en conclusion de cette dernière série d'articles qui donnent à penser ou à repenser les sociétés locales, L. Coupaye revient sur des questions théoriques liées à l'analyse des œuvres en prenant pour exemple un groupe qui n'était pas inclus dans l'exposition: les Abelam. Il analyse comment, ces dernières années, les travaux sur le statut des images et de leur interprétation, travaux qui ont été menés par Bateson et Gell, mais aussi Forge, Tuzin ou Kaufmann (pour ne nommer que quelques auteurs), partent d'un double constat à priori simple: il existe non seulement un hiatus entre l'image et son référent mais les artistes locaux se refusent à tout commentaire sur les signes ou les figures et quand, d'aventure, ils les nomment, ils y reconnaissent souvent chacun des entités différentes. Pour résoudre ce dernier point, naîtra petit à petit l'idée que l'instabilité des images répond à la nature changeante et imprévisible de la manifestation des ancêtres. Se pose alors la redoutable question de la nature de l'ancêtre et des formes de son apparition dans le monde des vivants par exemple sous la forme d'enveloppes - ce que sont les masques ou les sculptures. On est aussi en droit de se demander quel est le rôle des peintures corporelles dans cette manifestation - l'ancêtre ne s'incarnant que dans un corps peint. Comme l'auteur le remarque, ce n'est pas tant le corps comme première enveloppe qui importe que la peinture formant une seconde enveloppe. À l'image des poupées russes, ce système d'enveloppes peut être démultiplié, la maison des hommes devenant ainsi à son tour une enveloppe pour toutes les images qu'elle contient et donc un corps ancestral dont la puissance est inégalée. On ouvre ainsi à un jeu de mise en abyme redoutablement efficace qui permet de nombreux niveaux de lecture.Tous les travaux réunis ici sont, peu ou prou, redevables d'une longue histoire d'exploration, de collectes, de données de terrain et d'expositions. Une histoire marquée par la disparité de nos connaissances. Si certains groupes situés au nord de la rivière comme les Kwoma, les Abelam, les Boiken, ou ceux qui vivent dans la région marécageuse du moyen Sepik comme les Iatmul, les Chambri, les Sawos, sont connus, d'autres zones, comme la région en amont d'Ambunti, ou le bas Sepik, sont depuis longtemps peu explorées. Aussi déplorables que soit cette situation, on doit se résigner à ce que ces lacunes ne soient jamais comblées. À tout jamais, notre connaissance de ces sociétés dans leurs formes anciennes restera au mieux partielle, au pire inconnue. Ce constat ne doit cependant pas obérer des travaux contemporains sur le terrain ou sur les collections. Les articles réunis dans ce dossier en démontrent toute la richesse tout en posant un certain nombre d'axes et de problé- matiques de recherche. Il reste pourtant beaucoup à faire. Les collections n'ont été qu'exceptionnellement publiées, analysées et surtout comparées. Leur mise en ligne reste en-deçà de ce qu'elle devrait être.

Bien avant Margaret Mead, Reche notait que les cultures du Sepik se caractérisent par l'importation d'un groupe à un autre de biens matériels ou immatériels. Cette facilité d'absorber de nouveaux objets ou de nouveaux rituels est probablement un facteur important, si ce n'est fondateur, de la Sepikness. Or, les mécanismes et les effets de ces échanges sont peu décrits. Leur étude nécessiterait d'être affinée. Celle d'une série d'objets dont la date et le lieu de collecte sont connus permettrait d'établir une chronologie et de percevoir les variations au fil du temps. L'établissement de cette chronologie montrerait combien notre vision actuelle du Sepik reste statique.

Depuis l'origine de la découverte du Sepik, comme le montre le texte programmatique de von Luschan, la question de l'art occupe une place centrale dans les études sur la région. Cette place est due au fait que le Sepik fut reconnu comme un lieu de création d'une richesse exceptionnelle. Sans vouloir nier cette dimension, il ne faut pas oublier cependant que la culture matérielle nécessite une approche bien plus large. Une simple analyse des formes ne rend pas compte de la richesse des sens que l'étude de la matérialité des choses permet de comprendre. Comme le montrent plusieurs articles réunis ici, et pour reprendre une idée des "Instructions sommaires pour les collecteurs d'objets ethnographiques " ${ }^{6}$, les objets du quotidien sont, tout comme les objets techniques, détenteurs de sens. Ils peuvent être à l'origine d'une lecture aussi complexe que celle d'une figure d'ancêtre des maisons des hommes. Ce n'est pas là la moindre richesse du Sepik.

\section{BIBLIOGRAPHIE}

Bateson Gregory, 1971 (1936). La cérémonie du naven, Paris, Les éditions de Minuit.

Behrmann Walter, 1922. Im Stromgebiet des Sepik. Eine deutsche Forschungsreise in Neuguinea, Berlin, August Scherf.

-, 1917/1924. Der Sepik (Kaiserin-Augusta-Fluss) und sein Stromgebiet: geographischer Bericht der Kaiserin-Augusta-Fluss-Expedition 1912-13 auf der Insel Neuguinea, avec carte en 3 feuillets au $1 / 250000^{e}$, Berlin, Dietrich Reimer.

BüHLER Alfred, 1960. Kunststile am Sepik: Führer durch das Museum für Völkerkunde und Schweizerisches Museum für Volkskunde Basel, Sonderausstel-

6. Dans les Instructions (1931: 8-9), Marcel Griaule écrivait: "Les objets les plus communs sont ceux qui en apprennent le plus sur une civilisation. Une boîte de conserves, par exemple, caractérise mieux nos sociétés que le bijou le plus somptueux ou que le timbre le plus rare. Il ne faut donc pas craindre de recueillir les choses même les plus humbles et les plus méprisées. Un objet peut ne rien valoir à nos yeux non plus qu'aux yeux de l'indigène et être une inépuisable source de renseignements. (Exemple : les ustensiles de ménage, les cordes nouées pour un usage quelconque)». 
lung vom 11. Juni bis 30. Nov. 1960, Basel, Museum für Völkerkunde.

BüHLER Alfred und Paul Wirz, 1954. Kunstwerke vom Sepik: Sonderausstellung 1.10-31.12.1954, Museum für Völkerkunde und Schweizerische Museum für Volkskunde Basel.

Coiffier Christian (éd.), 2001. Le voyage de La Korrigane dans les mers du Sud, Paris, musée de l'Homme et Hazan édition.

Clark Alison and Nicholas Thomas (eds), 2017. Style and Meaning. Essays on the anthropology of art. Anthony Forge, Leiden, Sidestone Press, Pacific Presences 1.

Craig Barry, Ron Vanderwal and Christine WinTER, 2015. War Trophies or Curios: the War Museum Collection in Museum Victoria 1915-1920, Melbourne, Museum Victoria Publishing.

Forge Anthony, 1965. Art and Environment in the Sepik, in Prooceedings of the Royal Anthropological Institute of Great Britain and Ireland for 1965, London, Royal Anthropological Institute, pp. 23-31.

-, 2017 (1986). Draft Introduction to Sepik Culture History, the Proceedings from the second Wenner-Gren conference on Sepik Culture History 1986, Mijas, Spain, in A. Clark and N. Thomas (eds), Style and Meaning. Essays on the anthropology of art. Anthony Forge, Leiden, Sidestone Press, pp. 153-178.

Guiart Jean, 1968. Art de l'Océanie. Nouvelle-Guinée. Région Sepik, Paris, unesco et Albin Michel.

HaberLand Eike, 1968. The caves of Karawari. Catalogue d'exposition, New York, D’Arcy Gallery.

Haberland Eike und Meinhard Schuster, 1964. Sepik. Kunst aus Neuguinea. Aus den Sammlungen der Neuguinea-Expedition des Städt. Museums für Völkerkunde, Ausstellung im Städel'shen Kunstinstitut, Juni-August, Museums für Völkerkunde, Frankfurt am Main.

Houseman Michael et Carlo Severi, 1994. Naven ou Le donner à voir. Essai d'interprétation de l'action rituelle, Paris, CNRS et maison des sciences de l'homme.

Instructions sommaires pour les collecteurs d'objets ethnographiques, mission Dakar-Djibouti, mai 1931, Paris, Palais du Trocadéro, Musée d'ethnographie (http://gallica.bnf.fr/ark:/12148/bpt6k65415773).

Juillerat Bernard, 1993. La révocation des tambaran. Les Banaro de Richard Thurnwald revisités, Paris, CNRs Éd.

Kaufmann Christian, 2003. Korewori. Magic art of the Rain Forest, Basel, Museum der Kulturen.

-, 2017. Anthony Forge and Alfred Bühler: From Field Collecting to Friendship, in A. Clark and N. Thomas (eds), Style and Meaning. Essays on the anthropology of art. Anthony Forge, Leiden, Sidestone Press, pp. 181-207.

Kelm Heinz, 1966-1968. Kunst von Sepik, Berlin, Museum für Völkerkunde, 3 vols.

-, 1969. Kunstgegenstände aus dem Sepik-Gebiet, Baessler-Archiv N.F. xviI, pp. 303-364.

LutKeHaus Nancy L. et al. (eds), 1999. Sepik Heritage. Tradition and Change in Papua New Guinea, Durham N.V., Carolina Academic Press.

Mead Margaret, 1938. The Mountain Arapesh, Part 1. An importing culture, in Anthropological papers of the American Museum of Natural History 36, part 3.

Newton Douglas, 1971. Crocodile and Cassowary. Religious art of the Upper Sepik River, New Guinea, New York, The Museum of Primitive Art.

Reche Otto, 1913. Der Kaiserin-Augusta-Fluss, Ergebnisse der Südsee-Expedition 1908-1910, vol. 2A1, Hamburg, Friederichsen \& Co. Traduction anglaise par John Dennison, 2015. Otto Reche, The Empress Augusta / Sepik River, Otago, Departement of Anthropology and Archaeology, University of Otago.

Roesicke Adolf, 1914. Mitteilungen über ethnographische Ergebnisse der Kaiserin Augusta-Fluss-Expedition, Zeitschrift für Ethnologie 46, pp. 507-522.

Schindlbeck Markus, 2015. Unterwegs in der Südsee: AdolfRoesicke und seine Fahrten auf dem Sepik in Neuguinea, Berlin, Ethnologisches Museum \& Nicolai.

Schlaginhaufen Otto, 1910. Eine ethnographische Sammlung vom Kaiserin-Augustafluss in Neuguinea, Abhandlungen und Berichte des Königl. Zoologischen und Anthropologischen Museums zu Dresden XIII, part 2.

Schuster Meinhard, 1986. Symmetrische Formensprache auf Schilden aus dem Sepik-Gebiet, Symmetrie in Kunst, Natur und Wissenchaft, Mathildenhöhe Darmstadt. Ausstellung von 1. Juni bis 24. August 1986, Darmstadt, Band 1, pp. 129-136.

von Luschan Felix, 1911. Zur Ethnographie des Kaiserin-Augusta-Flusses, Baessler-Archiv 1, pp. 103-117.

Wardwell Allen, 1971. The Art of the Sepik, Catalogue d'exposition, The Art Institute of Chicago (16 octobre-18 novembre 1971), Chicago, Art Institute of Chicago.

Welsch Robert L. (ed.), 1998. An American Anthropologist in Melanesia: A.B. Lewis and the Joseph N. Field South Pacific expedition, 1909-1913, Honolulu, University of Hawai'i Press, 2 vols.

Wirz Paul, 1959. Kunst und Kult des Sepik Gebiets (Neu-Guinea), Koninklijk Institut voor de Tropen, KIT Mededeling 83 (Afdeling Cultureele en Physische Anthropologie 62). 